\title{
Análisis de la Arquitectura 5G en Relación a la Opción no Autónoma de la Asociación Global del Ecosistema Móvil (GSMA).
}

\author{
Narda Ortiz Campos \\ Profesor-Instituto Tecnológico Superior Luis Tello, Esmeraldas, (Ecuador) \\ njortiz@istluistello.edu.ec
}

RESUMEN

La quinta generación de tecnología móvil representa un avance significativo en la forma de comunicar los datos. El nuevo desarrollo económico de los países estará basado en esta nueva tecnología que ha llegado para transformar e innovar la forma tradicional de hacer las actividades cotidianas dentro del hogar, empresas, ciudades y gobiernos. El presente estudio tiene como objetivo analizar la arquitectura $5 \mathrm{G}$ en relación a la opción no autónoma de la Asociación Global del Ecosistema Móvil (GSMA). Para realizar el análisis se hizo un estudio sistemático de la literatura. Se utilizaron los métodos de análisis y síntesis y el deductivo e inductivo para presentar la información recolectada sobre la arquitectura 5G NSA, el estado actual de las telecomunicaciones en el Ecuador, y, las opciones de despliegue de la GSMA. Se descargo' información de sitios web oficiales de organizaciones como: GSMA, 5G Americas, ITU, 3GPPP, entre otras. De igual forma, se obtuvo información de artículos publicados en google Académico, Sciencie Direct, Scopus e IEEE. De todos los artículos encontrados solo 15 fueron seleccionados. Como resultado se obtuvo que la 5G NSA es uno de el mejor escenario que tienen las operadoras móviles del Ecuador para el despliegue de la 5G. Finalmente, a través del análisis se pudo determinar que la operadora móvil CONECEL S.A. es la que tiene mayor cantidad de radiobases con tecnología 4G desplegadas en el país, por lo tanto; tiene mayores probabilidades de tener una alta cobertura $5 \mathrm{G}$ cuando realice el despliegue de esta tecnología.

Palabras clave: $5 \mathrm{G} ; \mathrm{NSA}$; arquitectura $5 \mathrm{G}$.

\begin{abstract}
The fifth generation of mobile technology represents a significant advance in the way data is communicated. The new economic development of countries will be based on this new technology that has arrived to transform and innovate the traditional way of doing everyday activities within the home, businesses, cities and governments. The present study aims to analyze the 5G architecture in relation to the nonautonomous option of the Global Mobile Ecosystem Association (GSMA). To perform the analysis, a systematic literature survey was conducted. The methods of analysis and synthesis and deductive and inductive were used to present the information collected on the 5G NSA architecture, the current state of telecommunications in Ecuador, and, the GSMA deployment options. Information was downloaded from official websites of organizations such as: GSMA, 5G Americas, ITU, 3GPPP, among others. Similarly, information was obtained from articles published in Google Scholar, Sciencie Direct, Scopus and IEEE. Of all the articles found, only 19 were selected. As a result, it was obtained that the 5G NSA is one of the best scenarios that mobile operators in Ecuador have for the deployment of 5G. Finally, through the analysis it was determined that the mobile operator CONECEL S.A. is the one that has the largest number of radio bases with $4 \mathrm{G}$ technology deployed in the country, therefore, it is more likely to have a high $5 \mathrm{G}$ coverage when deploying this technology.
\end{abstract}

Keywords: 5G; NSA; 5 G architecture. 


\section{Introducción}

La quinta generación de tecnología móvil representa un avance significativo en la forma de comunicar los datos. 5G va mucho más allá de los teléfonos inteligentes, pues es la tecnología que dará inicio a una transformación digital profunda de la sociedad y de la economía de los países, las soluciones vinculadas al Internet de las cosas y el big data, la robótica, la realidad virtual o la ultra alta definición, alcanzaran su despliegue e implantación sobre redes 5G (J, Cerezo y P, 2014).

El nuevo desarrollo económico de los países estará basado en esta nueva tecnología que ha llegado para transformar e innovar la forma tradicional de hacer las actividades cotidianas dentro del hogar, empresas, ciudades y gobiernos. La 5G representa para los países la oportunidad de crear una nación digitalizada en donde todas las tareas podrán hacerse a través de un clic desde cualquier lugar del mundo. Actualmente, existen dos escenarios para implementar la $5 \mathrm{G}$ en los países, éstas son la opción 2 conocida como independiente o autónoma cuyas siglas en inglés son SA, y la opción 3 no independiente o no autónoma de siglas NSA. Adicionalmente, la GSMA ha elaborado tres variaciones para SA denominadas opción 2 y 5; y, para la NSA las opciones 3,4 y 7 (GSMA, 2020; Berisha, 2021).

El escenario de despliegue NSA permite reutilizar la infraestructura de la 4G utilizando una conexión dual al EPC, esta característica es una ventaja que ha brindado facilidad en la implementación de la 5G (GSMA, 2018). En la actualidad, más de 90 países han desplegado la red $5 \mathrm{G}$ y en los últimos años se han desarrollado algunos estudios sobre esta tecnología. Así, en relación a la forma de implementación y descripción de la arquitectura 5G NSA los autores (Yuan et al., 2020) han elaborado una investigación sobre el método de planificación mixto de $5 G$ y LTE bajo el modelo de arquitectura NSA; mientras que, en el estudio de (Suthar et al., 2020) se proporciona arquitecturas y métodos de interfuncionamiento para la migración de las tecnologías $4 \mathrm{G}$ y $5 \mathrm{G}$ evidenciando los desafíos claves del despliegue; de la misma forma, (Dubey and Meena, 2020) investigaron sobre la mejora del rendimiento mediante conectividad dual en redes $5 \mathrm{G}$ nueva radio no independiente, entre otros. 
Asimismo, existen muchos informes técnicos que abordan el tema del despliegue de la $5 \mathrm{G}$ desde la perspectiva de Gemalto los operadores móviles deberán implementar la red $5 G$ y mantener operativa la red $4 G$. Adicionalmente, deberán operar nuevos espectros lo que implica inversiones fuertes en la infraestructura de la red (Gemalto, 2016). De la misma forma, GSMA detalla las opciones de despliegue bajo el escenario NSA elaborados por la asociación de participación de tercera generación (3GPP) que permitirán la coexistencia de las redes 4G y 5G; y, la 3GPP en el "release" 15 detalla la arquitectura, espectro, protocolos, entre otros aspectos técnicos relevantes para que la cuarta y quinta generación puedan interrelacionar sus funciones 3GPP (2018). También, la (International Telecommunication Union (ITU), 2019) presenta las consideraciones técnicas para la implementación de la 5G basado en el "release" 15.

Ecuador no ha podido avanzar en sus iniciativas del despliegue de la $5 G$ según (Ministerio de Telecomunicaciones y de la Sociedad de la Información (Mintel), 2020), las razones son: la inexistencia de una hoja de ruta, la carencia de un marco regulatorio y la ausencia de políticas públicas para la implementación de 5G. De igual forma, el estado no ha podido realizar la subasta para ofertar a las operadoras móviles el espectro radioeléctrico de 3.5$3.8 \mathrm{GHz}$, el cual permitirá que las operadoras puedan brindar a sus usuarios la tecnología $5 G$.

De aquí en adelante este proyecto está organizado de la siguiente manera. En la Sección 2, se presenta los trabajos relacionados. En la Sección 3, se expone la metodología. En la Sección 4, se exhibe los resultados obtenidos. En la Sección 5, se reportan la discusión. Finalmente, en la Sección 6, se presentan las conclusiones.

\section{Trabajos relacionados.}

En esta sección se presentan algunos trabajos relacionados con la presente investigación. Los cuales se enfocan en la migración hacia la 5G. Un aspecto importante para migrar a la $5 G$ es encontrar una respuesta a cómo; por qué y dónde debe desplegar las tecnologías o integrarlas en la red existente (Zakeri et al., 2020).

La (GSMA, 2018), en su informe camino a la 5G establece 6 rutas o estrategias de migración que podrían emplear las operadoras móviles para el despliegue de la 5G. Tres de las seis rutas se enfocan en el escenario NSA. El estudio indica que la industria de la telefonía móvil debe respetar la necesidad de los diferentes operadores de seguir diferentes estrategias de migración con algún grado de divergencia. Este trabajo contribuye con el análisis de cada una de las rutas bajo las siguientes perspectivas: viabilidad del caso de uso, consideraciones de despliegue, el impacto de la etapa de migración en el dispositivo y de la red, y, el impacto en el servicio de voz.

Del mismo modo, (Zakeri et al., 2020), estableció estrategias de migración hacia la red 5G desde la perspectiva del E2E. Este documento presenta una visión práctica y completa sobre cómo migrar al $5 \mathrm{G}$. En él se muestra y discute diferentes vías de migración de la red de acceso a la radio (RAN), la red de transporte (TN), la red central (CN) considerando factores, como el e coste, el tráfico de datos local/nacional, la comercialización. De igual forma, la investigación aporta con un plan a largo plazo para la evolución de la red de 4G - $5 \mathrm{G}$ y de $5 \mathrm{G}$ a $6 \mathrm{G}$. 
Asimismo, (Suthar et al., 2020), en su investigación sobre la migración e interfuncionamiento entre $4 \mathrm{G}$ y $5 \mathrm{G}$ proporcionan estrategias para el interfuncionamiento y la migración entre las tecnologías móviles 4G y $5 \mathrm{G}$ para ello, los autores identificaron diferentes escenarios de interfuncionamiento, los desafíos asociados, y abordaron sus posibles soluciones. De esta forma, el estudio posee varias contribuciones, está son: 1) la opción de despliegue 3X NSA es las estrategias preferida por los operadores móviles para la el paso de los servicios 4G a los 5G; 2)el NSA tiene un alto impacto en el EPC y que la migración debe ser evolutiva del NSA-SA; $y, 3$ ) las operadoras deben considerar una interoperabilidad sin interrupciones del núcleo de red $5 \mathrm{G}$ con el núcleo de paquetes $4 \mathrm{G}$.

También (Unión Internacional de Telecomunicaciones, 2018), analizó las estrategias que deben realizar los actores de la formulación de políticas en los países para impulsar la transición de los operadores inalámbricos para actualizar sus redes de 4G a 5G, especialmente en zonas urbanas, donde es probable que se priorice la instalación de estas últimas. Este informe presenta un enfoque diferente a los anteriores debido a que proporciona las estrategias de migración que deberán poner en práctica los gobiernos y las operadoras móviles para impulsar la implantación de las redes 5G. Algunas de las estrategias son; revisar sus normativas sobre política digital y realizar inversiones para el despliegue de la $5 \mathrm{G}$ con cautela considerando para ello los requerimientos de la industria.

\section{Metodología}

El presente proyecto tiene un enfoque cualitativo. Este estudio es bibliográfico, se realizó una búsqueda sistemática de la literatura existente sobre la arquitectura 5G NSA y las rutas de las opciones de despliegue de la GSMA con el propósito de establecer la ruta que mejor se adapte a la realidad del país.

En la presente investigación se establecieron y respondieron tres interrogantes (RQs) y para las RQ1 Y RQ3 se estableció los siguientes protocolos de búsqueda:5G Architecture AND NSA y 5G AND NSA. Sin embargo, para la RQ2 se estableció el protocolo: "Telecomunicaciones AND Ecuador".

Para la revisión bibliográfica se descargó información de sitios web oficiales de organizaciones internacionales que apoyan el despliegue de la 5G como: GSMA, ITU, 3GPPP, entre otras. De igual forma, se obtuvo información de artículos publicados en Google Académico, Sciencie Direct, Scopus e IEEE. En la Figura 1 se puede observar el número de artículos que se encontraron al aplicar los protocolos de búsqueda.

Para la revisión bibliográfica se descargó información de sitios web oficiales de organizaciones internacionales que apoyan el despliegue de la 5G como: GSMA, ITU, 3GPPP, entre otras. De igual forma, se obtuvo información de artículos publicados en Google Académico, Sciencie Direct, Scopus e IEEE. En la tabla 2 se puede observar el número de artículos que se encontraron al aplicar los protocolos de búsqueda. 


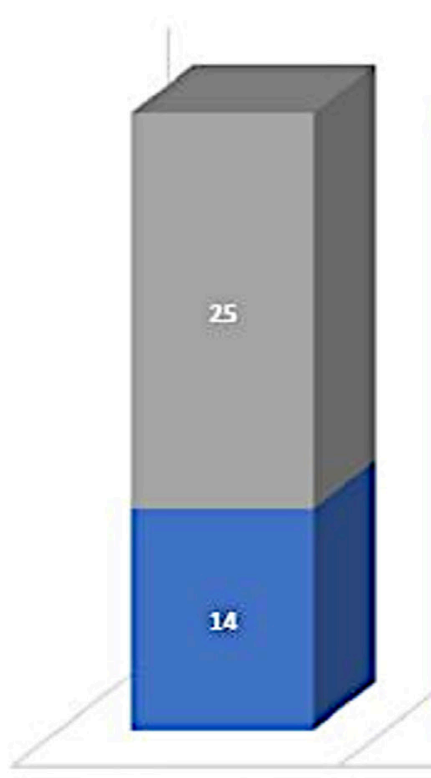

IEEEXPLORE

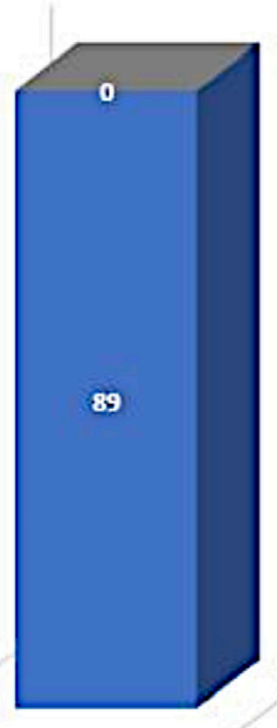

scopus
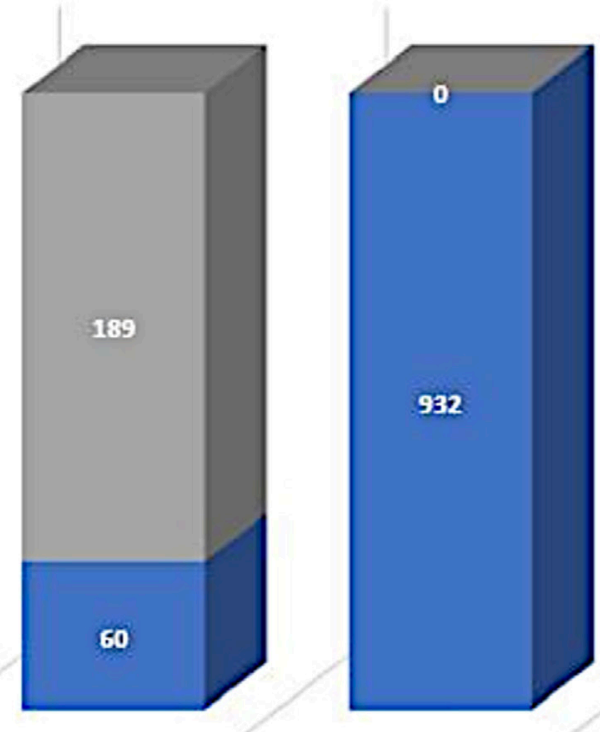

GOOGLE SCHOLAR

Fig. 1. Número de artículos encontrados por protocolos de Búsqueda Relacionados con la $5 G$ NSA.

Los artículos encontrados fueron filtrados utilizando los siguientes criterios de inclusión: 1) fecha máxima de publicación de 5 años, es decir; desde el 2017-2021; 2) que el idioma de los estudios fuera en inglés o español; 3) que la información proviniera de sitios web oficiales de organizaciones e instituciones especializadas en el despliegue de la 5G; y, 4) que los documentos tuvieran información sobre el despliegue de la NSA 5G y estado de las telecomunicaciones en Ecuador. No se consideraron aquellos artículos en los que se evidenció que no contenían información relacionada a las tres interrogantes planteadas en esta investigación. Después de aplicar los filtros fueron seleccionadas 15 publicaciones. En la Tabla 3 se puede observar lar referencias bibliográficas seleccionadas.

\begin{tabular}{|c|c|}
\hline \# Id & Referencias Bibliográficas \\
\hline T1 & (ARCOTEL (Agencia de Regulación y Control de las Telecomunicaciones), 2020) \\
\hline T2 & (ARCOTEL (Agencia de Regulación y Control de las Telecomunicaciones), 2021) \\
\hline T3 & (Berisha, 2021) \\
\hline T4 & (GSMA, 2018) \\
\hline T5 & (GSMA, 2020) \\
\hline T6 & (Hequan, 2020) \\
\hline T7 & (3GPP, 2018) \\
\hline T8 & (Peñafiel Yanza and Chenche Villacís, 2015) \\
\hline T9 & (Rommer et al., 2020a) \\
\hline T10 & (Rommer et al., 2020b) \\
\hline T11 & (Samaniego-Moncayo et al., 2020) \\
\hline T12 & (Suthar et al., 2020) \\
\hline T13 & (Udoh and Srivastava, 2020) \\
\hline T14 & (Yuan et al., 2020) \\
\hline T15 & (Zapata et al., 2020) \\
\hline
\end{tabular}

Tabla 1. Referencias bibliográficas seleccionadas 
Una vez seleccionados los documentos se almacenaron en el gestor bibliográfico Mendeley para facilitar el proceso de ordenar, seleccionar y citar la información. Luego los artículos seleccionados se almacenados en el software QDA miner para realizar el análisis cualitativo. En QDA Miner se creó por cada pregunta de investigación una categoría, así como sus respectivos códigos. En la Figura 2 se puede evidenciar un ejemplo de la frecuencia de los códigos en los documentos que fueron analizados para responder la RQ1.

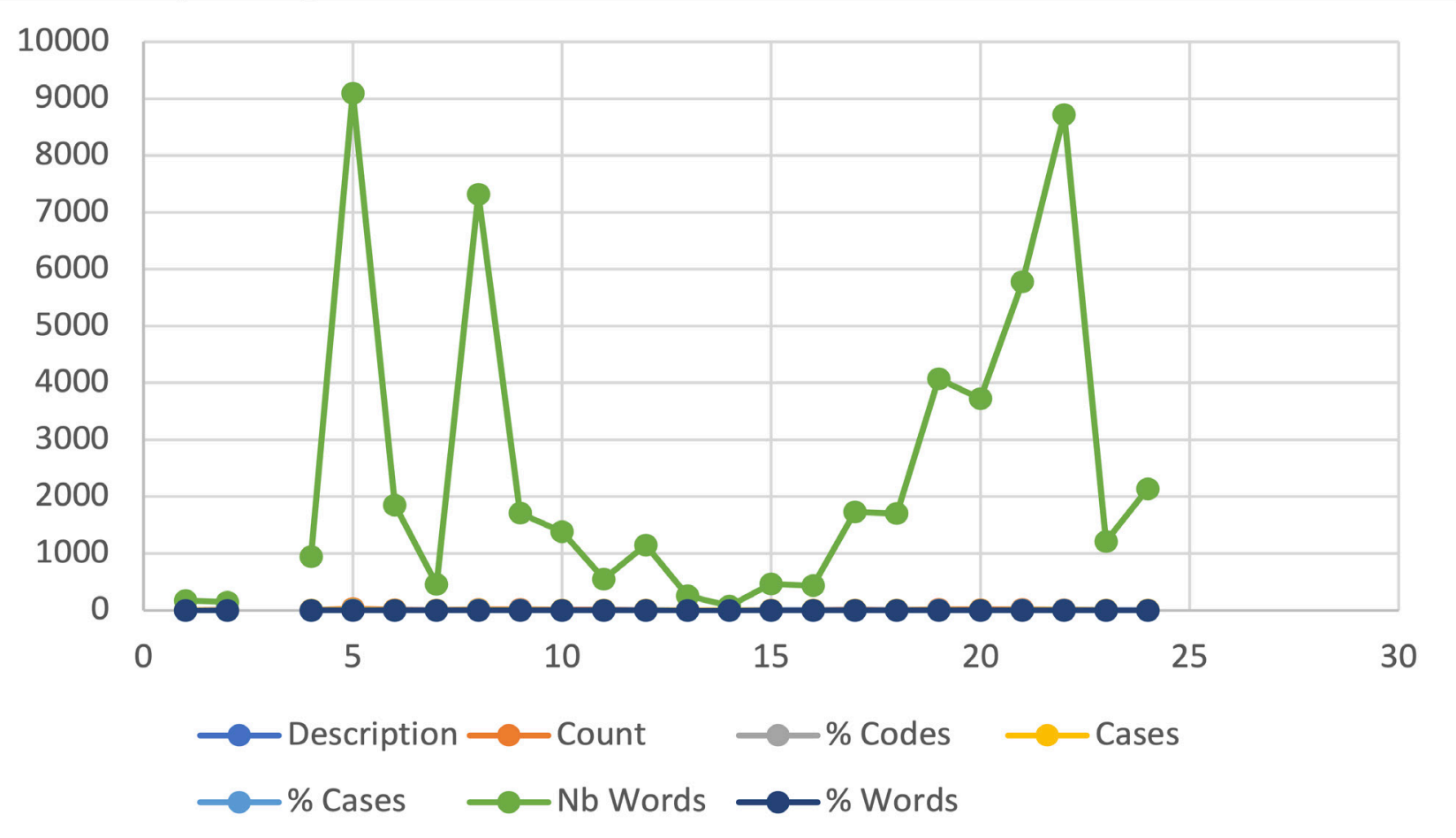

Fig. 2. frecuencia d ellos códigos para analizar la RQ1

\section{Resultados}

El análisis de la arquitectura $5 \mathrm{G}$ en relación a la opción no autónoma de la asociación global del ecosistema móvil (GSMA), se concibió a través de la resolución de las siguientes interrogantes.

\section{RQ1. ¿Cuáles son los componentes de la arquitectura 5G NSA?}

A nivel mundial las operadoras de servicios móviles han realizado sus despliegues $5 \mathrm{G}$ basados en el escenario NSA, excepto los chinos (Hequan, 2020; Udoh and Srivastava, 2020). La" opción 3x" del 3GPP es la opción más elegida por los operadores para el despliegue de la 5G NSA (Yuan et al., 2020). El modo de red de la opción 3 se refiere a la arquitectura de conexión dual de LTE y/o NR al EPC (GSMA, 2018), en la que la red de acceso $5 \mathrm{G}$ se conecta a la red básica 4G; es decir, la 5G NSA reutiliza la infraestructura de la tecnología4G para realizar el rápido despliegue de la 5G (Berisha, 2021; Hequan, 2020; Yuan et al., 2020; Udoh and Srivastava, 2020). La 5G NSA es la fase de despliegue inicial que afrontara' la convergencia de la arquitectura de red, la señalización y las capacidades de edición de la próxima generación de los sistemas de telefonía móvil (Berisha, 2021). Por medio de las redes centrales $4 G$ existentes y las nuevas estaciones base $5 G$ la NSA logra aumentar la capacidad de banda ancha móvil mejorado (eMBB) para los datos en los dispositivos 5G (Hequan, 2020; Udoh and Srivastava, 2020). Sin embargo, se emplearán la infraestructura de la cuarta generación para la comunicación de voz. 
Según (3GPP, 2018; Rommer et al., 2020a), al escenario de despliegue NSA también se le conoce como EN-DC, que significa conectividad dual entre $4 G$ y $5 G$; es decir, que la $E$ hace referencia a la tecnología $4 G$ en relación a los eNB y el acceso a la radio $4 G$. La $N$ indica la tecnología de la NR. Y, DC son las siglas que significan la conectividad dual. Para que el diseño EN-DC tenga éxito, la radio $4 G$ se utilizara' para transportar la señalización de control, mientras que la NR y/o la LTE se utilizarán para los datos del usuario (GSMA, 2020).

En la arquitectura NSA (Figura 3) la estación base de la NR (nodo lógico en-gNB) se conecta a la estación base del 4G LTE (nodo lógico eNB) a través de la interfaz X2. Dado que el acceso a la red de $5 \mathrm{G}$ se despliega conjuntamente del sistema LTE, tanto la conectividad 4G como la 5G están disponibles (3GPP, 2018).

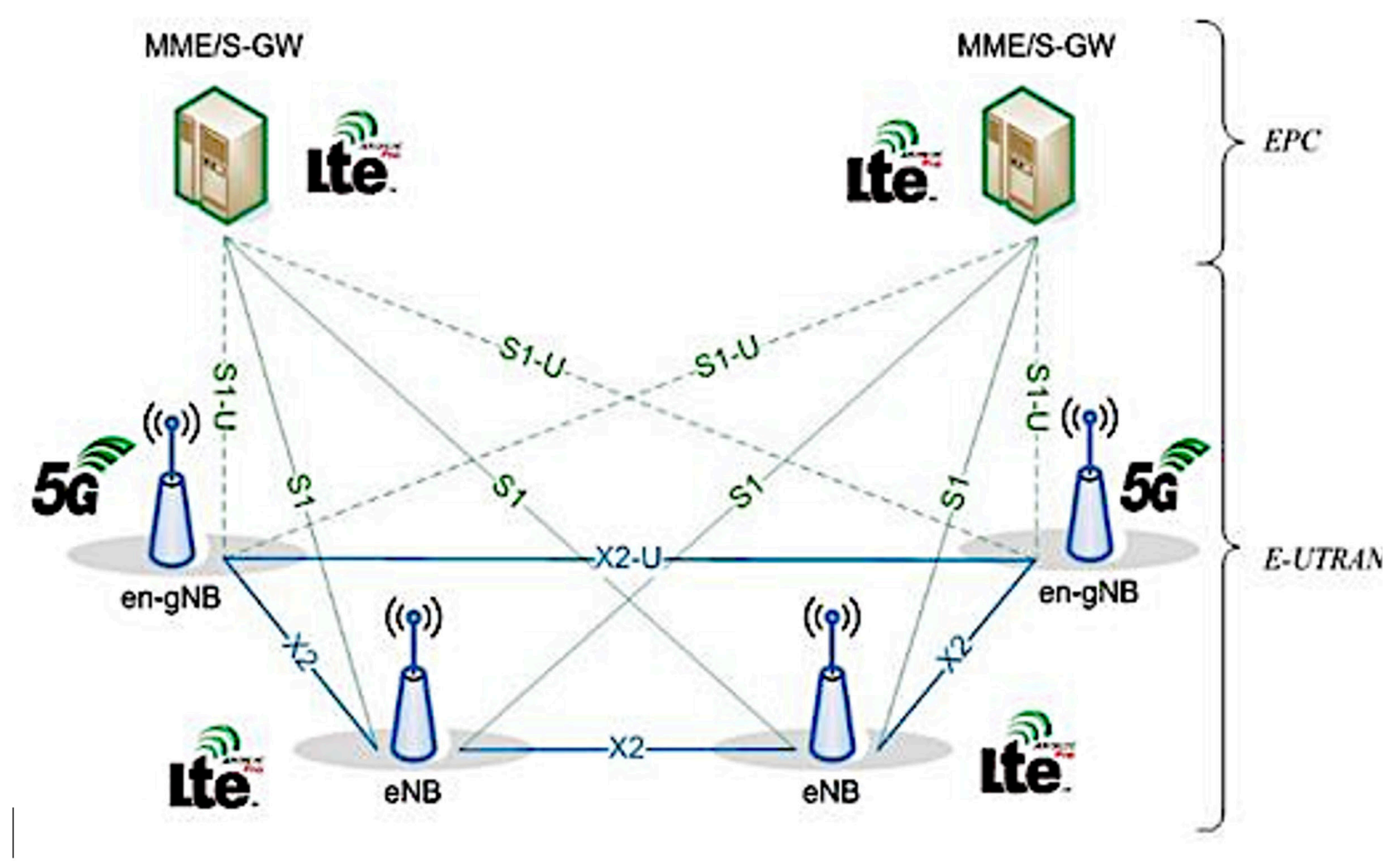

Fig. 3. Arquitectura 5G NSA.

La red de acceso radioeléctrico E-UTRAN esta' compuesta por el eNB que es el nodo maestro (MN) mientras que el en-gNB es el nodo secundario, y X2 es la interfaz que utilizan para la comunicación entre los nodos. El MME es la entidad de gestión de la movilidad responsable de la conectividad de la señalización del plano de control con el MN, este componente es responsable de la conectividad de los UE (Rommer et al., 2020b). Por cada portador EPS, la E-UTRAN decide si se utiliza un NM o un SN. Por ejemplo, el eNB maestro puede decidir utilizar el engNB 5G para los datos de usuario (la conexión PDN del Internet), para disfrutar del aspecto de alta capacidad de la 5G, y utilizar el eNB 4G para la señalización IMS y la voz IMS, para disfrutar del aspecto de capa de cobertura robusta de la 4G (3GPP, 2018). 
La 3GPP ha denominado Nueva Radio (NR) a la nueva tecnología de radio de la 5G. La NR consiste en varias bases radioeléctricas conectadas a la red central como entre sí. La NR se basa en varios conceptos tecnológicos de LTE como son el esquema de modulación OFDM y multiplexación TDD/FD; sin embargo, la NR ha ampliado esos conceptos para garantizar la flexibilidad en la comunicación. El despliegue de la NR para una amplia gama de frecuencias diferentes es posible gracias a una estructura muy flexible de la capa física (Rommer et al., 2020b). Adicionalmente, la NR suele combinar las técnicas MIMO y Beamforming para cumplir con algunos de los requisitos de muy alta capacidad y altas velocidades de datos para servicios $5 \mathrm{G}$.

El EPC fue diseñado para tener funciones de plano de control separadas, por lo tanto; la función de la gestión de la movilidad fue separada de las funciones de gestión de la sesión con la gestión del plano de usuario (Rommer et al., 2020a). En la (GSMA, 2020), se establece como funciones del EPC la gestión de la movilidad, gestión de la sesión, uso compartido de la red, separación del plano de control y de usuario control y usuario, control de políticas y tarificación, y, gestión de suscripciones y seguridad.

Según (Hequan, 2020), existen dos escenarios típicos para la actualización de EPC para admitir la implementación de 5G. En el escenario A: el EPC físico se actualiza para admitir NSA; y la expansión de la capacidad se basa en el EPC físico. En el escenario B: se crea una nueva red EPC virtualizada para admitir la NSA de forma independiente; se establece la interoperabilidad entre el nuevo EPC virtualizado y el EPC físico, y, la expansión de la capacidad se basa en el EPC virtualizado. Adicionalmente, para actualizar el EPC se debe considerar la comparación de las opciones 3/3a/3x, la estrategia de actualización de la red central 4G y la actualización de las funciones de la red (3GPP, 2018).

\section{RQ2. ¿Cuál es el estado actual de las telecomunicaciones en Ecuador?}

El servicio móvil avanzado (SMA) sostiene su funcionamiento a través del uso de las redes móviles. La evolución de las redes móviles está dada por la 1G, 2G,3G, 4G y 5G (Samaniego-Moncayo et al., 2020); sin embargo, en el Ecuador solo se ha implementado las redes móviles inferiores a la 5G. Cada una de los cuatros generaciones han tenido su correspondencia con los siguientes estándares de comunicación AMS; GSM - CDMA; UTMS; y LTE. Según (ARCOTEL(Agencia de Regulación y Control de las Telecomunicaciones), 2020) los servicios proporcionados por la $1 \mathrm{G}$ fueron solo de voz a velocidades de $1 \mathrm{kbps}$ a 2,4 kbps, y utilizó tecnología análoga. Caso contrario, la 2G,3G y 4G brindan servicios con tecnología digital a velocidades de 14kbps a $64 \mathrm{Kbps}$; 384KBPS a 2Mbps; y $100 \mathrm{Mbps}$ a 1 Gbps respectivamente. De hecho, a partir de la $2 \mathrm{G}$ las redes móviles ofrecieron servicios de voz digital y SMS; aunque solo la 3 G y $4 G$ han brindado servicios de internet limitado y ancho de banda mejorada correspondientemente.

En Ecuador la cobertura de SMA es de alrededor de $94 \%$ a nivel poblacional. La cobertura SMA dependerá' de la infraestructura de telecomunicaciones instalada por parte de las operadoras móviles en la zona o 'área del país que se necesite cubrir (Zapata et al., 2020). En el país, existe al menos una estación base de las tecnologías $2 \mathrm{G}, 3 \mathrm{G}$ y $4 \mathrm{G}$ en las parroquias del Ecuador. Así se establece que la 2G cubre 620 parroquias; la 3G esta' presente en 625 parroquias; y la 4G existe en 388 parroquias; es decir que hay un total de 676 estaciones bases instaladas a nivel nacional, lo que equivale una cobertura del $65 \%$ de las parroquias ecuatorianas. Las provincias con mayor estaciones bases instaladas son: Pichincha, Guayas, Manabí y Azuay (ARCOTEL(Agencia de Regulación y Control de las Telecomunicaciones), 
2020). Es evidente que las estaciones bases con tecnología4G tienen una menor cobertura, a pesar de que 'estas son relevantes para el despliegue de la $5 G$ a través del escenario NSA.

Actualmente, en Ecuador existen tres operadoras móviles estas son CONOCEL S. A.; OTECEL S. A y CNT EP su participación en el mercado es de 51,50\%; 30,82\% y 17,68\% respectivamente (ARCOTEL (Agencia de Regulación y Control de las Telecomunicaciones), 2021). De las operadoras móviles existentes en el país CNT EP es la única operadora móvil estatal que ofrece sus servicios a los ciudadanos a partir del año 2013. (Peñafiel Yanza and Chenche Villacís, 2015), detallan que en el país son las operadoras móviles las encargadas de instalar su infraestructura en las áreas geográficas que desean cubrir para ofrecer los servicios de telefonía.

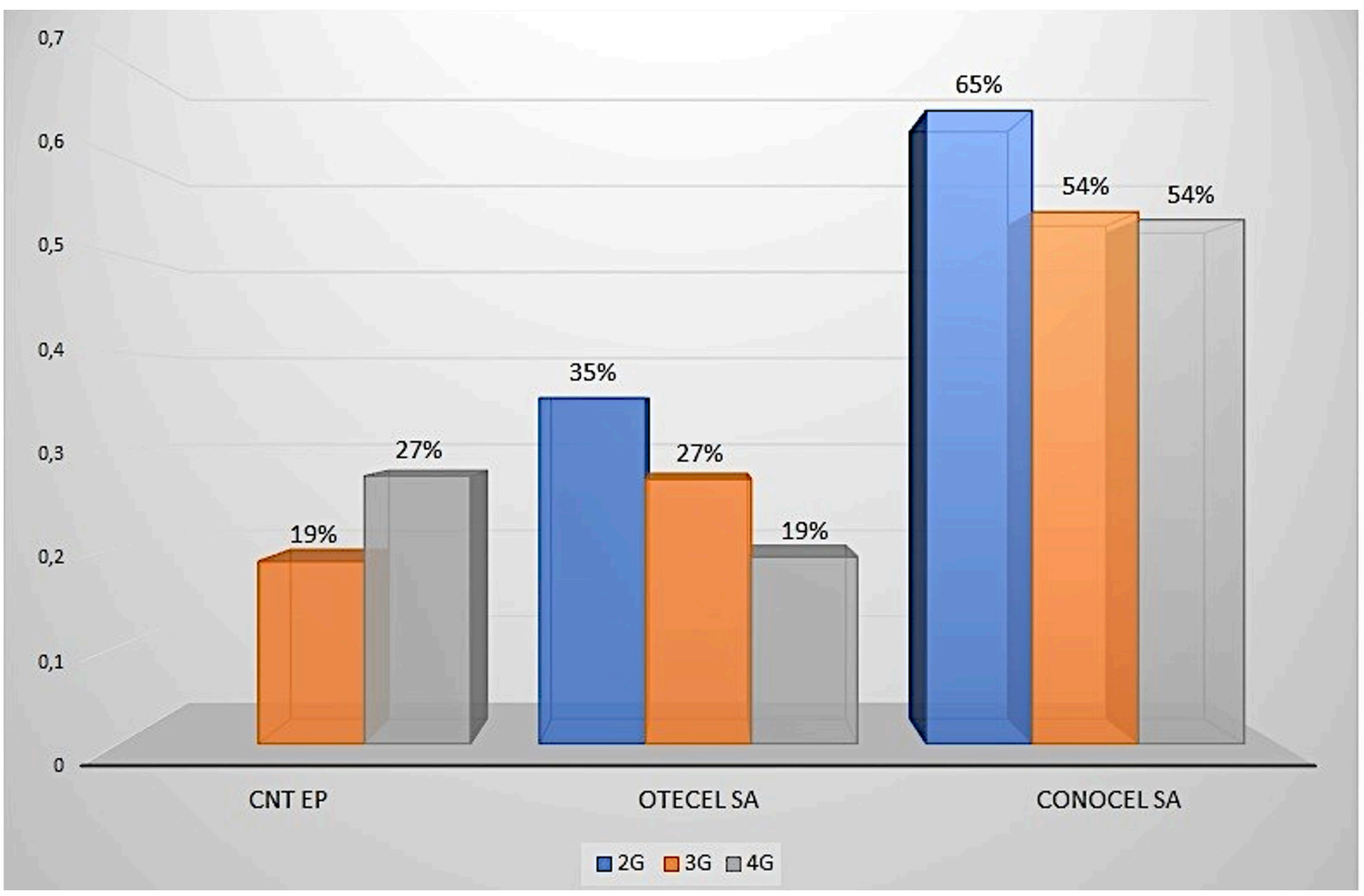

Fig. 4. Estaciones bases instaladas por las operadoras

(ARCOTEL (Agencia de Regulación y Control de las Telecomunicaciones), 2021)

Según (ARCOTEL (Agencia de Regulación y Control de las Telecomunicaciones), 2021), el total de estaciones bases instaladas por cada una de las operadoras es de 3.838 (CNT EP); 5195 (OTECEL S. A.); y 11.367 (CONECEL S. A.). En la figura 3 se puede apreciar que OTECEL S. A. y CONOCEL S. A. ofrecen servicios con las tecnologías 2G, 3G y 4G; sin embargo, CNT EP, no brinda servicios con tecnologías 2G. De igual forma, se puede observar que si bien el crecimiento de la tecnología4G de OTECEL S. A. ha sido menor en porcentaje con relación a CNT EP la cantidad total de estaciones bases de OTECEL S. A. es mayor, permitiendo ofrecer una mejor señal a los habitantes del Ecuador, cubriendo más zonas con las tecnologías $2 \mathrm{G}$ y $3 \mathrm{G}$. Asimismo, CONOCEL S. A. posee el mayor despliegue pues esta operadora tiene la mayor cantidad de estaciones bases en todo el país lo que le permite tener una mayor cobertura. Además, en esa figura se evidencia que OTECEL S. A. y CNT EP lideran el despliegue de la 4G; es decir; que el 37\% de las estaciones bases a nivel nacional pertenecen a esa tecnología. 
El espectro es un recurso que administra el estado, 'este es un bien intangible, natural y limitado el cual es necesario para que las redes de telefonía móvil puedan transmitir datos y brindar un servicio eficiente a sus usuarios. El espectro se asigna a las operadoras móviles por diferentes bandas de frecuencia. (Zapata et al., 2020), señalan que el Ecuador tiene asignado para a los servicios de telecomunicaciones móviles internacionales (IMT, por sus siglas en inglés) $1.040 \mathrm{MHz}$, de los cuales solo $280 \mathrm{MHZ}$ es el espectro efectivo asignado. Las operadoras móviles en el país tienen estipulada las siguientes bandas: CONECEL S. A. tiene $95 \mathrm{MHz}$ en las bandas de $850 \mathrm{MHz}(25 \mathrm{MHz}), 1900 \mathrm{MHz}(30 \mathrm{MHz})$ y $1700-2100 \mathrm{MHz}$ $(40 \mathrm{MHz})$. OTECEL S. A. mantiene $85 \mathrm{MHz}$ distribuidos en las bandas de $850 \mathrm{MHz}(25 \mathrm{MHz})$ y $1900 \mathrm{MHz}(60 \mathrm{MHz})$, mientras que en el caso de CNT EP se registran $100 \mathrm{MHz}$ en las bandas de $700 \mathrm{MHz}(30 \mathrm{MHz}), 1900 \mathrm{MHz}(30 \mathrm{MHz}), 1700-2100 \mathrm{MHz}(40 \mathrm{MHz})$. La banda $3.5 \mathrm{GHz}$ esta asignación para servicios fijos-móviles, sin embargo, 'esta es un recurso necesario para la transición de $4 \mathrm{G}$ a $5 \mathrm{G}$.

\section{RQ3. ¿Qué ruta que debe seguir los operadores móviles en Ecuador para la implementación de la 5G}

(GSMA, 2018), ha presentado tres opciones de despliegue en relación del escenario NSA, estas son: opción 3) utilizando EPC y un eNB LTE que es el nodo maestro y el en-gNB NR es el nodo; opción 4) utilizando 5GC y un en-gNB NR actuando como maestro y gNB LTE como el nodo secundario; y, opción7) usando 5GC y un ng-eNB como nodo maestro y un gNB actúa como nodo secundario. En la Figura 5 se observan dichas opciones.
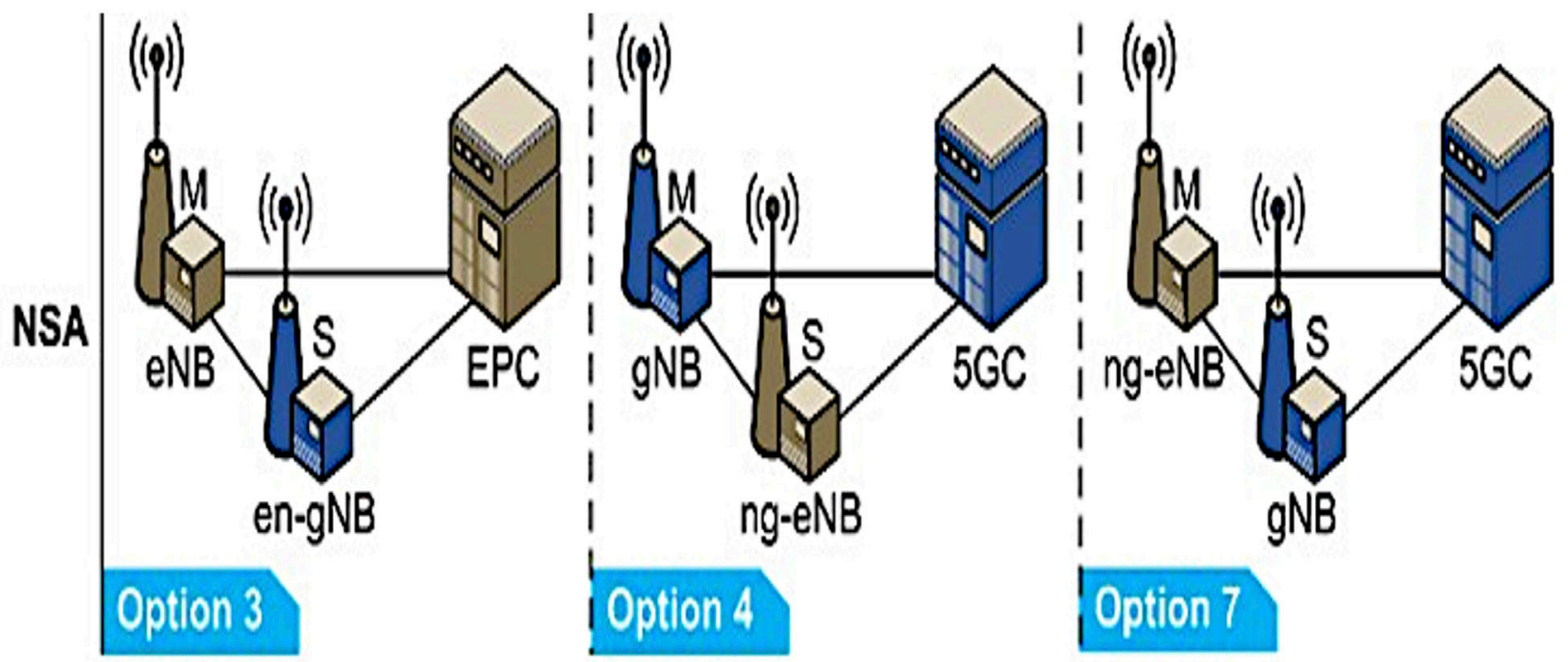

Fig. 5. Opciones de despliegue NSA.

Cada una de las opciones tienen subopciones de despliegue, es así que, la opción3 tiene las subopciones 3/3a/3x; la opción $4-4 / 4 a$; y, la opción $7-7 / 7 a / 7 x$. La diferencia en cada una de las subopciones está dada por las variantes en la conexión del plano de usuario y/o del plano de control. En (Suthar et al., 2020), se encuentra una explicación detallada de las subopciones. (GSMA, 2019), realizó una entrevista a operadoras y grupos de operadoras para establecer las preferencias que tenían estos en relaciona las opciones que utilizarían para el despliegue de la $5 \mathrm{G}$ y tuvo como resultado que las operadoras móviles consideran para sus planes de despliegue el usar las opciones 4, 5 y 7. Lo antes mencionado demuestra la tendencia de las operadoras móviles por el uso del escenario NSA para la primera fase del despliegue de la tecnología5G. Para evidenciar los requerimientos y cambios en la infraestructura de red que las operadoras móviles tendrán que realizar en las opciones 3 y 
7 la organización (GSMA, 2018), ha elaborado una comparación técnica. En la Figura 6 se puede observar información referente a: las especificaciones de la 3GPP, espectro, núcleo de red, fragmentación de la red y QoS 5G, aprovechamiento de LTE, actualización de LTE, interfuncionamiento de la RAN, sesión de datos inter-RAT y compatibilidad con SA de todas las opciones del NSA.

\begin{tabular}{|l|l|l|}
\hline & OPCIÓN 3 & OPCIÓN 7 \\
\hline Especificación 3GPP 5G & Release 15 (2017) & Release 15 (2018) \\
\hline Espectro 5G & $\begin{array}{l}\text { Sub-6GHz y } \\
\text { mmWave }\end{array}$ & $\begin{array}{l}\text { Sub-6GHz y } \\
\text { mmWave }\end{array}$ \\
\hline CN & EPC & 5GC \\
\hline $\begin{array}{l}\text { Interfuncionamiento Con } \\
\text { la CN }\end{array}$ & No requiere & No requiere \\
\hline $\begin{array}{l}\text { Fragmentación de la red y } \\
\text { QoS 5G }\end{array}$ & No se admite & Se admite \\
\hline $\begin{array}{l}\text { Impacto en la UE para el } \\
\text { modo dual }\end{array}$ & (5G/LTE) EPC-NAS & 5GC/EPC-NAS \\
\hline Aprovechamiento de LTE & Completo & Completo \\
\hline Actualización de LTE & $\begin{array}{l}\text { Necesario (eNB y } \\
\text { EPC) }\end{array}$ & $\begin{array}{l}\text { Necesario } \\
\text { eNB y 5GC) }\end{array}$ \\
\hline Interfuncionamiento de la \\
RAN & EN-DC & NGEN-DC \\
\hline Sesión de datos inter-RAT & $\begin{array}{l}\text { MR-DC e } \\
\text { intrasistema }\end{array}$ & $\begin{array}{l}\text { MR-DC e } \\
\text { intrasistema }\end{array}$ \\
\hline Compatibilidad con SA & Bajo & Medio \\
\hline
\end{tabular}

Fig. 6. Comparación técnica entre las opciones 3 y 7 del NSA Fuente: (GSMA, 2018).

Existen diferentes rutas o caminos que las operadoras móviles pueden seguir para su despliegue de la 5G. Esto se debe a que 5G puede ser desplegada usando el 5GC o EPC. Por tal razón, los miembros del proyecto introducción a la $5 \mathrm{G}$ creen que existe una mayor posibilidad en que las operadoras móviles realicen las rutas detalladas en la Figura 7 . El análisis de las rutas considera en: 1) casos de uso eMBB, mloT, URLLC: la capacidad de abordar los mismos: 2) dispositivos: apoyo de los primeros dispositivos 5G, núcleo de red y espectro en la etapa de migración; 3) despliegue/red: se considera el impacto en la etapa de migración en los dispositivos y en la red; $y, 4)$ voz: hace referencia a la continuidad del servicio que se ofrece por el paso de migración (GSMA, 2018). 


\begin{tabular}{|c|c|c|c|c|}
\hline Ruta & Uso de Caso & Dispositivo & Despliegue/Red & Voz \\
\hline EPS a SA\#2 & Casos de uso de $5 \mathrm{G}$ completos & $\begin{array}{l}\text {-5G Beneficios básicos } \\
\text { - Necesita retener } \\
\text { EPC }\end{array}$ & $\begin{array}{l}\text { - Poco impacto en } \\
4 \mathrm{G} \\
\text { - Sistema } \quad 4 \mathrm{G} / 5 \mathrm{G} \\
\text { interworking requerido }\end{array}$ & $\begin{array}{l}\text { - Voz IMS } \\
\text { - No CS interviniendo desde } \\
5 \text { GS }\end{array}$ \\
\hline EPS a NSA\#3 & $\begin{array}{l}\text { - Apoyo limitado para el caso de uso } \\
\text { de } 5 G \text { (en relación al eMBBS) }\end{array}$ & $\begin{array}{l}\text { - Aprovechar el LTE } \\
\text { - Rápido tiempo de } \\
\text { comercialización } \\
\text { - No beneficios del Core 5G }\end{array}$ & $\begin{array}{l}\text { - Procedimientos EPC } \\
\text { - Impacto en } 4 G\end{array}$ & $\begin{array}{l}\text { - Aprovechar las ventajas } \\
\text { existentes } \\
\text {-Servicio de VoLTE }\end{array}$ \\
\hline $\begin{array}{l}\text { NSA\#3 a } \\
\text { NSA\#7/SA\#5 }\end{array}$ & $\begin{array}{l}\text { - Casos de uso de } 5 \mathrm{G} \text { completos } \\
\text { - Inicialmente limitado }\end{array}$ & $\begin{array}{l}\text {-5G Beneficios básicos } \\
\text { - Necesita retener EPC }\end{array}$ & $\begin{array}{l}\text { - Impacto en NR, LTE } \\
\text { - Impacto en el IMS } \\
\text { - Despliegue de 5GC }\end{array}$ & $\begin{array}{l}\text { - Voz IMS } \\
\text { - No CS interviniendo desde } \\
5 \mathrm{GS}\end{array}$ \\
\hline $\begin{array}{l}\mathrm{NSA} \# 3 \text { a } \\
\mathrm{NSA} \# 3 / \mathrm{SA} \# 2\end{array}$ & $\begin{array}{l}\text { - Casos de uso de } 5 \mathrm{G} \text { completos } \\
\text { - Inicialmente limitado } \\
\text {-Migración del núcleo }\end{array}$ & $\begin{array}{l}\text { - 5G Beneficios básicos } \\
\text { - Necesita retener EPC } \\
\text {-NR de área amplia }\end{array}$ & $\begin{array}{l}\text { - Impacto en NR, LTE } \\
\text { - Impacto en el IMS } \\
\text { - Despliegue de 5GC }\end{array}$ & $\begin{array}{l}\text { - Voz IMS } \\
\text { - No CS interviniendo desde } \\
5 G S\end{array}$ \\
\hline $\begin{array}{l}\text { NSA\#3 a } \\
\text { NSA\#4/SA\#2 }\end{array}$ & $\begin{array}{l}\text { - Casos de uso de } 5 \mathrm{G} \text { completos } \\
\text { - Inicialmente limitado } \\
\text { - Migración del núcleo }\end{array}$ & $\begin{array}{l}\text {-5G Beneficios básicos } \\
\text { - Necesita retener EPC }\end{array}$ & $\begin{array}{l}\text { - Impacto en NR, LTE } \\
\text { - Impacto en el IMS } \\
\text { - Despliegue de 5GC }\end{array}$ & $\begin{array}{l}\text { - Voz IMS } \\
\text { - No CS interviniendo desde } \\
\text { 5GS }\end{array}$ \\
\hline
\end{tabular}

Fig. 7. Rutas para el despliegue de la 5 G (GSMA, 2018).

(Suthar et al., 2020), exponen las rutas de transiciones de 4G a $5 \mathrm{G}$ más populares adoptadas por la mayoría de las operadoras móviles, estas son: opción1 LTE a opción 7x NSA a opción2 SA; opción1 LTE a opción3x NSA a opción2 SA; y, opción 1 LTE a opción4 NSA a opción2 SA. En tal sentido, Ecuador puede utilizar una ruta cuya primera fase de despliegue sea NSA. De igual forma, el análisis que se realizó en la figura 5 permite determinar que la perspectiva del despliegue de red en la ruta EPS a NSA\#3 es la que mejor se acopla al país. Esto se debe a que las operadoras móviles en Ecuador no tienen el rango de espectro requerido para la $5 G$ y el estado no tiene políticas claras para apoyar el despliegue de esta nueva tecnología. Adicionalmente, esta ruta presenta las siguientes ventajas: capacidad de desplegar la nueva radio mientras se ancla la comunicación al EPC; aprovecha el EPC; permite mantener la Fiberización de los usuarios; servicio de VoLTE; entre otros (GSMA, 2018).

Las operadoras móviles del país podrán considerar la guía detallada que presenta (GSMA, 2018) para el despliegue de la $5 G$ en el escenario NSA. También podrá tomar como referencia el estudio de (Yuan et al., 2020), en el cual se presenta la estructura del plan general que pueden seguir las operadoras móviles para el despliegue de la 5G. Este plan contempla: 1) la planificación comercial; 2) planificadores de indicadores de negocio; 3) planificadores de frecuencia; y 4) planificadores de la capacidad. 


\section{Discusión}

La 5G NSA es el mejor escenario que tienen las operadoras móviles del Ecuador para el despliegue de la 5G, sin embargo; los usuarios no podrán gozar de todos los casos de uso de esta nueva tecnología móvil. De igual forma, (GSMA, 2018) explica que las capacidades el EPC puede ser un cuello de botella para los casos de uso de la 5G, en tal sentido, es importante el despliegue de la 5G SA. Además, existen varios problemas en la implementación de la red 5G a través del escenario NSA. (Dubey and Meena, 2020) y (Suthar et al., 2020) expresan que se debe mejorar el escenario de movilidad de usuario de la arquitectura NSA. (GSMA, 2020) manifiesta que el intercambio de infraestructura entre las operadoras móviles será un problema que surgirá por lo complejo que es compartir equipos de red en forma activa. Asimismo, GSMA dice que el cambio de infraestructura LTE puede ser un reto para las operadoras.

\section{Conclusiones.}

A nivel mundial los operadores móviles han utilizado el escenario NSA para el despliegue inicial de la 5G, debido a que se apoya en la arquitectura LTE. A través del análisis se pudo determinar que la operadora móvil CONECEL S.A. es la que tiene mayor cantidad de radiobases con tecnología4G desplegadas en el país, por lo tanto, tiene mayores probabilidades de tener una alta cobertura 5G cuando realice el despliegue de esta tecnología. Previo al despliegue la operadora debe realizar una planificación pues así lo establecen Shutar y yuan 2020 al explicar que para el despliegue de la 5G debe existir una planificación a largo plazo y continua que permita implementar el despliegue de la 5G de forma tal que evite el desperdicio de recursos de la red y los costos de inversión.

La arquitectura 5G NSA presente muchas facilidades para la implementación de la 5G, pues permite la reutilización de la infraestructura de la 4G, sin embargo, las operadoras móviles deberán adquirir nuevas tecnologías y frecuencias de radio que les permitan diseñar una red $5 \mathrm{G}$ flexive, es decir; las redes deberán tolerar el aumento en el tráfico de los nuevos servicios que surgirán de los casos de uso de la 5G. El rol de las operadoras móviles en el despliegue de la $5 \mathrm{G}$ será el de potencializar la arquitectura de la red en la nube; acondicionar el espectro; preparar la infraestructura informática y de redes; entre toras. El gobierno del Ecuador también deberá contribuir para la implementación de la 5G a través de la revisión y adaptación de las normativas relacionadas al desarrollo de las TIC.

\section{Bibliografía}

3GPP. 3GPP TS 22.278 V15.4.0. Technical report, 2018. URL http://www.3gpp.org.

ARCOTEL (Agencia de Regulación y Control de las Telecomunicaciones). Servicio de Acceso a Internet Fija. Technical report, 2020.

ARCOTEL (Agencia de Regulación y Control de las Telecomunicaciones). Servicio Móvil Avanzado, 2021.

D. Berisha. 5G SA and NSA Solutions. PhD thesis, 2021.

S. Dubey and J. Meena. Improvement of throughput using dual connectivity in nonstandalone 5G NR networks. Proceedings of the 3rd International Conference on Smart Systems and Inventive Technology, ICSSIT 2020, (Icssit):1196\{1200, 2020. doi: 
10.1109/ICSSIT48917.2020.9214179.

Gemalto. Presentación de las redes 5G Características y usos. Technical report, 2016.

GSMA. Road to 5G : Introduction and Migration. Technical Report April, 2018. URL https://www.gsma.com/futurenetworks/wp-content/uploads/2018/04/Road-to-5GIntroduction-and-Migration_FINAL.pdf.

GSMA. 5G Implementation Guidelines. Technical Report July, 2019. URL https://www.gsma. com/futurenetworks/wp-content/uploads/2019/03/5G-Implementation-Guidelinev2.0-July-2019.pdf.

GSMA. 5G Implementation Guidelines : NSA Option 3. Technical Report February, 2020. URL https://www.gsma.com/futurenetworks/wiki/5g-implementation-guidelines/.

W. U. Hequan. Ten Reections on 5 G. 18(1):1\{4, 2020. doi: 10.12142/ZTECOM. 202001001.

International Telecommunication Union (ITU). 5G networks and 3GPP Release 15. Technical Report October, 2019.

C. J, Cerezo y P. El impacto de 5G. Technical report, 2014. URL http://evocaimagen.com/ cuadernos-tecnologia/cuadernos-evoca-tecnologia-1.pdf.

Ministerio de Telecomunicaciones y de la Sociedad de la Informaci_on (Mintel).El Ministro Michelena destaca los bene_cios que traer_a al Ecuador la tecnolog_a 5G \{ Ministerio de Telecomunicaciones y de la Sociedad de la Informaci_on, 2020. URL https:// www.telecomunicaciones.gob.ec/el-ministro-michelena-destaca-los-beneficios-quetraera-al-ecuador-la-tecnologia-5g/

L. E. Peña_el Yanza and F.ís@. La convivencia de la tecnología 4G con 3G y 2G en Ecuador. Investigativo, 6(6):15\{37, 2015. ISSN 13906399. doi: 10.31095/investigatio.2015.6.1.

S. Rommer, P. Hedman, M. Olsson, L. Frid, S. Sultana, and C. Mulligan. Dual connectivity. In $5 G$ Core Networks, number Dc, pages 265\{285. 2020a. ISBN 9780081030097. doi: 10.1016/b978-0-08-103009-7.00012-0.

S. Rommer, P. Hedman, M. Olsson, L. Frid, S. Sultana, and C. Mulligan. Architecture overview. In 5G Core Networks, pages 15\{72. 2020b. ISBN 9780081030097. doi: 10.1016/b978-0-08-103009-7.00003-x.

B. Samaniego-Moncayo, J. Herrera-Tapia, J. P. Ponce, J._on-Varela@, and P. Henr_quezCoronel. An_alisis del despliegue y uso de la tecnolog_a celular en Ecuador. Associa_c ao lb_erica de Sistemas e Tecnologias de Informacao, 29:51\{66, 2020.

P. Suthar, V. Agarwal, R. S. Shetty, and A. Jangam. Migration and Interworking between 4G and 5G. 2020 IEEE 3rd 5G World Forum, 5GWF 2020 - Conference Proceedings, pages 401\{406, 2020. doi: 10.1109/5GWF49715.2020.9221021.

S. J. Udoh and V. M. Srivastava. Analytical modeling of radio network performance for $5 G$ (Non-standalone) and it's network connectivity. Journal of Communications, 15(12):886\{895, 2020. ISSN 17962021. doi: 10.12720/jcm.15.12.886-895. 
Uni_on Internacional de Telecomunicaciones. Sentando las bases para la 5G: Oportunidades y desaf_os. Technical report, 2018. URL https://www.itu.int/dms_pub/itu-d/opb/ pref/D-PREF-BB.5G_01-2018-PDF-S.pdf.

Q. Yuan, Q. Qian, Y. Mo, and H. Chen. Research on mixed planning method of $5 \mathrm{G}$ and LTE. Proceedings-3rd International Conference on Informationand ComputerTechnologies, ICICT 2020, pages 489\{493, 2020. doi: 10.1109/ICICT50521.2020.00084.

A.Zakeri, N. Gholipoor, M. Tajallifar, S. Ebrahimi, M. R. Javan, N. Mokari, and

R. Sharafat. E2E Migration Strategies Towards 5G: Long-term Migration

Plan and Evolution Roadmap. pages 1\{10, 2020. ISSN 2331-8422. URL http://arxiv.org/ $\mathrm{abs} / 2002.08984$.

R. Zapata, E. I. Rodríguez, and A. G. Zaballos. Estado actual de las telecomunicaciones y la banda ancha en Ecuador. Technical report, 2020. URL https://publications.iadb. 


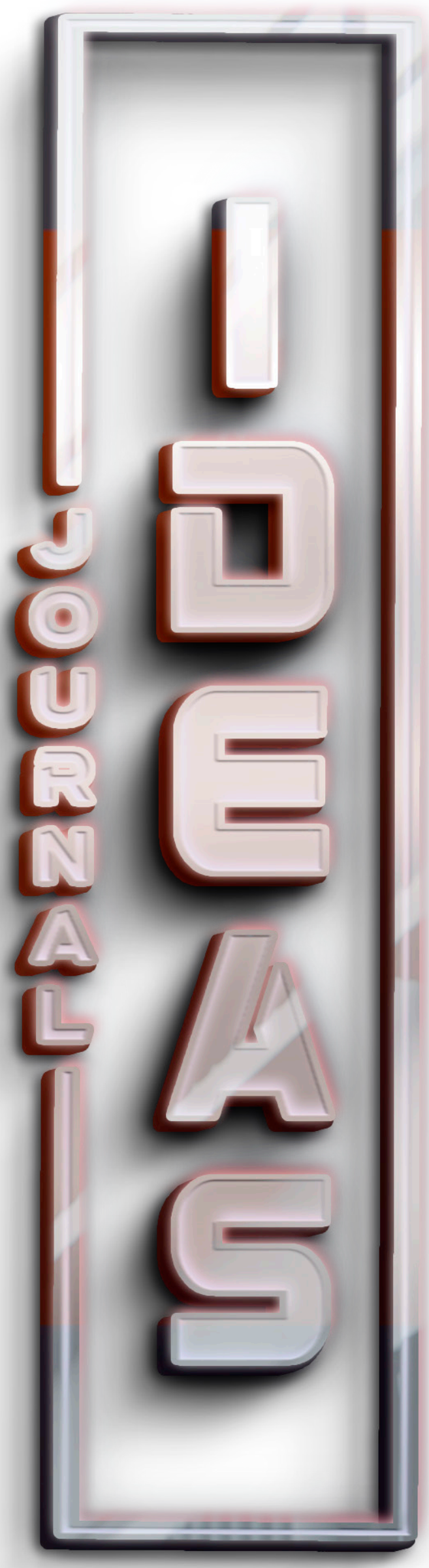

